\title{
Black hole ringdown as a probe for dark energy
}

\author{
Johannes Noller, ${ }^{1,2}$ Luca Santoni $\odot,{ }^{3}$ Enrico Trincherini $\odot,{ }^{4,5}$ and Leonardo G. Trombetta $\oplus^{4,5}$ \\ ${ }^{1}$ Institute for Theoretical Studies, ETH Zürich, Clausiusstrasse 47, 8092 Zürich, Switzerland \\ ${ }^{2}$ Institute for Particle Physics and Astrophysics, ETH Zürich, 8093 Zürich, Switzerland \\ ${ }^{3}$ Department of Physics, Center for Theoretical Physics, Columbia University, 538 West 120th Street, \\ New York, New York 10027, USA \\ ${ }^{4}$ Scuola Normale Superiore, Piazza dei Cavalieri 7, 56126 Pisa, Italy \\ ${ }^{5}$ INFN-Sezione di Pisa, 56100 Pisa, Italy
}

(Received 14 March 2020; accepted 3 April 2020; published 23 April 2020)

\begin{abstract}
Under the assumption that a dynamical scalar field is responsible for the current acceleration of the Universe, we explore the possibility of probing its physics in black hole merger processes with gravitational wave interferometers. Remaining agnostic about the microscopic physics, we use an effective field theory approach to describe the scalar dynamics. We investigate the case in which some of the higher-derivative operators, that are highly suppressed on cosmological scales, instead become important on typical distances for black holes. If a coupling to the Gauss-Bonnet operator is one of them, a nontrivial background profile for the scalar field can be sourced in the surroundings of the black hole, resulting in a potentially large amount of "hair." In turn, this can induce sizeable modifications to the spacetime geometry or a mixing between the scalar and the gravitational perturbations. Both effects will ultimately translate into a modification of the quasinormal mode spectrum in a way that is also sensitive to other operators besides the one sourcing the scalar background. The presence of deviations from the predictions of general relativity in the observed spectrum can therefore serve as a window onto dark energy physics.
\end{abstract}

DOI: 10.1103/PhysRevD.101.084049

\section{INTRODUCTION}

The direct detection of gravitational radiation has marked the birth of gravitational wave astronomy, opening up the possibility to explore the Universe via a new fundamental messenger [1]. This new possibility not only allows us to access the regimes of strong gravity in astronomical systems, but it will also have profound consequences for cosmology. Along this line, in the present work, we will address the following question: under the assumption that the current acceleration of the Universe is driven by a dynamical scalar sector, to what extent can its physics be probed by looking at the signals emitted in a black hole coalescence process? Indeed, the presence of a black hole can induce a large pileup effect of the scalar profile in its surroundings, enhancing the field's nonlinearities. This means that some of the Lagrangian operators that provide negligible contributions to the cosmological background may become dominant near the black hole, potentially leaving signatures on the emitted gravitational waves.

For this to be possible, the strong restrictions on the presence of nontrivial scalar profiles around static, spherically symmetric black holes must be overcome. These are usually phrased in terms of so-called no-hair theorems (for a review see e.g., Refs. [2,3]). Therefore, in order for the scalar background to be nonzero in the first place and consequently affect the black hole dynamics, the theory must belong to the class of exceptions to such theorems. ${ }^{1}$ The simplest and most studied example to evade the no-hair restrictions has been the case of a linear coupling between the scalar and the Gauss-Bonnet operator (sGB) [6]. In the literature, such a shift-symmetric operator has so far only been considered in the minimalistic setting in which the only other operator in the Lagrangian for the scalar is the canonical kinetic term [7-13]. In this paper, we will extend this setup substantially. Motivated by our assumption that a shift-symmetric scalar field accounts for the dark energy component of the Universe on cosmological scales, we will consider scalar Lagrangians where, together with the kinetic term and the coupling to Gauss-Bonnet, a very general set of operators is included and study if at least one of them becomes large in the vicinity of a black hole. When this happens, as we discuss below, it has several novel important consequences. Just to mention two, we will show that both the theoretical [7] and observational [13] upper bounds on the coupling of the scalar Gauss-Bonnet operator can be relaxed by the presence of an additional

\footnotetext{
${ }^{1}$ Note that perturbations around the black hole can be affected by the scalar even when its background is vanishing; see e.g., Refs. [4,5]. Here, we will focus on black holes with nontrivial scalar field backgrounds.
} 
term like the cubic Galileon [14], broadening the range of values that such a coupling can take. Moreover, even though the scalar background is sourced by a single operator, at the Schwarzschild radius, at least one extra operator becomes of comparable size, opening up a wider spectrum of potentially observable signatures that can serve as a window onto dark energy physics.

We structure the paper as follows. In Sec. II, we introduce a class of shift-symmetric effective field theories (EFT) that can be responsible for the accelerated expansion of the Universe, and we characterize its relevant energy scales. Then, we show how the recent measurement of the speed of gravitational waves affects these models. In Sec. III, we discuss the sGB operator that must be present in the EFT to source a background scalar profile around black holes, and we estimate the size of the hair. In Sec. IV, we derive two classes of observable effects in the ringdown phase due to the dark energy field, namely, deviations from the Schwarzschild geometry and kinetic mixing of metric and scalar perturbations. In Sec. V, we describe the current most stringent bounds on the size of the hair coming from precision tests of gravity at different length scales. Section VI contains explicit examples of dark energy models that are consistent with those bounds and, at the same time, give observable effects in a binary black hole coalescence. Finally, Sec. VII contains our conclusions.

\section{SETUP}

Remaining agnostic about the microscopic theory of the dark sector, we will parametrize the scalar dynamics following an effective field theory perspective. The very general class of (shift-symmetric) dark energy models we will consider in this paper, which was introduced in Ref. [15], is schematically defined by a scalar Lagrangian with two energy scales $[L$ is a function with $O(1)$ dimensionless parameters $\left.{ }^{2}\right]$,

$$
\mathcal{L}=\Lambda_{2}^{4} L\left(\frac{(\partial \phi)^{2}}{\Lambda_{2}^{4}}, \frac{\nabla^{2} \phi}{\Lambda_{3}^{3}}\right)
$$

where $\Lambda_{3}$ is the UV cutoff of the effective theory and $\Lambda_{2} \gg \Lambda_{3}$, together with $M_{\mathrm{P}}$, is associated with the explicit breaking of the Galileon symmetry $\partial_{\mu} \phi \rightarrow$ $\partial_{\mu} \phi+b_{\mu}$ [14]. The values of these two energy scales are usually chosen to be such that operators with one derivative per field and the leading higher-derivative (HD) ones, all belonging to the Horndeski class [16], are similarly responsible for the accelerated expansion of the Universe on cosmological distances. For a timedependent background of the scalar field $\phi_{0}(t)$ in an

\footnotetext{
${ }^{2}$ Throughout the paper, we are assuming that couplings are $O(1)$, and we do not write them explicitly; we also omit factors of $4 \pi$ in all the estimates for simplicity.
}

Friedmann-Robertson-Walker (FRW) geometry defined by the Hubble parameter $H(t)$, this assumption implies that $\Lambda_{2}^{4}$ is the energy density of the Universe today and therefore $\Lambda_{2}=\left(M_{\mathrm{P}} H_{0}\right)^{1 / 2} \sim 10^{7} \mathrm{~km}^{-1}$ while $\Lambda_{3}=$ $\left(M_{\mathrm{P}} H_{0}^{2}\right)^{1 / 3} \sim 10^{-3} \mathrm{~km}^{-1}$. In this way, indeed, on the cosmological solution, both $X_{0} \equiv\left(\partial \phi_{0}\right)^{2} / \Lambda_{2}^{4}$ and $Z_{0} \equiv$ $\nabla^{2} \phi_{0} / \Lambda_{3}^{3} \sim H_{0} \partial \phi_{0} / \Lambda_{3}^{3}$ are of $O(1)$.

The large hierarchy - 10 orders of magnitude — between $\Lambda_{2}$ and $\Lambda_{3}$ is necessary to enhance the effect of HD operators up to the point of making them comparable, at the present horizon scale $H_{0}^{-1}$, with the ones that depend only on the first derivative of the scalar field. Such an extreme regime, which is nevertheless radiatively stable thanks to the approximate Galileon symmetry [15,17], is mainly motivated by phenomenological reasons: it is the one that allows for the largest variety of potentially observable signatures in the large scale structure.

The presence of higher-derivative operators at the scale $\Lambda_{3}$ in the dark energy models described by (1) is already being strongly constrained by the extraordinarily precise measurement of the speed of gravitational waves, made possible by the observation of the neutron star merger event GW170817 and of its electromagnetic counterpart GRB 170817A [18]. ${ }^{3}$ Consider for example the two types of operators that are present in the EFT (1) that are usually called quartic and quintic Horndeski. ${ }^{4}$ When evaluated on the FRW background, they can affect the speed of propagation of gravity, $c_{T}$ :

$$
\begin{aligned}
\frac{(\partial \phi)^{2}\left(\nabla^{2} \phi\right)^{2}}{\Lambda_{3}^{6}} & \rightarrow \frac{\left(\partial \phi_{0}\right)^{4}}{\Lambda_{3}^{6} M_{\mathrm{P}}{ }^{2}}(\partial h)^{2} \\
\frac{(\partial \phi)^{2}\left(\nabla^{2} \phi\right)^{3}}{\Lambda_{3}^{9}} & \rightarrow \frac{\left(\partial \phi_{0}\right)^{4}}{\Lambda_{3}^{6} M_{\mathrm{P}}{ }^{2}} \frac{\partial^{2} \phi_{0}}{\Lambda_{3}^{3}}(\partial h)^{2} ;
\end{aligned}
$$

if such operators play a role in the cosmological evolution, which requires as we discussed $\Lambda_{3}^{3} M_{\mathrm{P}} \sim M_{\mathrm{P}}^{2} H_{0}^{2}$, their contribution to $c_{T}$ is of $O(1)$.

One way to make the theory consistent with the bound $\left|c_{T}^{2}-1\right| \leq 10^{-15}$ is then to assume that the coefficients of all the different operators giving rise to deviations in the speed of propagation of gravitational waves form luminality are extremely small [20-23]. We will instead assume that the UV cutoff of the dark energy EFT, and therefore the characteristic scale of the higher-derivative operators, is larger than $\left(M_{\mathrm{P}} H_{0}^{2}\right)^{1 / 3} \sim 10^{-3} \mathrm{~km}^{-1}$. Let $\Lambda>\Lambda_{3}$ be this new scale:

\footnotetext{
${ }^{3}$ Note that the frequencies of the LIGO measurement of GW170817 are close to $\Lambda_{3}$, so additional assumptions about the UV physics are implicitly made when using this measurement to constrain such "cosmological" operators suppressed by $\Lambda_{3}$ [19].

${ }^{4}$ The exact definition of the operators is given below in Eq. (6). Here, we are just keeping track schematically of the number of fields and derivatives.
} 


$\begin{array}{cccc}\Lambda_{3} & \longmapsto & 10^{5} \Lambda_{3} & \Lambda_{2}=10^{10} \Lambda_{3} \\ 10^{-3} \mathrm{~km}^{-1} & \text { deviation from } & \text { satisfies } & 10^{7} \mathrm{~km}^{-1} \\ \mathrm{HD} \mathscr{O}(1) & c_{T}=1 \text { smaller } & \text { amplitudes } & \text { conventional } \\ \text { on cosmology } & \text { than } 10^{-15} & \text { bound } & \text { shift-symmetric } \\ & & & \text { EFT }\end{array}$

FIG. 1. The hierarchy of scales for $\Lambda$ (3) and effects of setting $\Lambda$ to the respective scale.

$$
\mathcal{L}=\Lambda_{2}^{4} L\left(\frac{(\partial \phi)^{2}}{\Lambda_{2}^{4}}, \frac{\nabla^{2} \phi}{\Lambda^{3}}\right)
$$

clearly, the contribution to $c_{T}^{2}$ from the two classes of operators in (2) in this case will be reduced respectively by factors $\left(\Lambda_{3} / \Lambda\right)^{6}$ and $\left(\Lambda_{3} / \Lambda\right)^{9}$. As a result, it is enough that $\Lambda>10^{3} \Lambda_{3}$ for the theory to be in agreement with observations, by which we mean not only the bound on $\left|c_{T}^{2}-1\right|$ but also the constraints on graviton decay [24] and dark energy instabilities induced by gravitational waves [25].

There is another independent, and more fundamental, motivation to consider larger values of the scale $\Lambda$. It is well known that general properties of the S-matrix, unitarity, analyticity, and crossing symmetry, imply positivity bounds for amplitudes at low energies, which in turn constrain the coefficients of EFT operators [26]. When these bounds are applied to theories with weakly broken Galileon invariance [15], they imply that the separation between symmetry-breaking and symmetrypreserving operators cannot be too large while keeping the UV cutoff fixed [27] (see Refs. [28-30] for closely related prior work). More specifically, in the case of the Lagrangian (3), the condition becomes

$$
\Lambda_{\mathrm{UV}} \lesssim\left(\Lambda / \Lambda_{2}\right)^{3 / 2} 10^{7} \mathrm{~km}^{-1}
$$

This is to say that, if one separates the scale $\Lambda$ too much from the symmetry-breaking scale $\Lambda_{2}$, i.e., when the ratio $\Lambda / \Lambda_{2}$ is taken to be very small, then the new degrees of freedom associated with a UV completion (that respects the basic principles mentioned above) must enter at energies $\Lambda_{\mathrm{UV}}<\Lambda$, therefore reducing the regime of validity of the EFT.

As it should be clear from the previous discussion, once the scale $\Lambda$ is taken to be parametrically larger than $\left(10^{3} \mathrm{~km}\right)^{-1}$, there will be no sizable effect on the cosmological evolution from HD operators. From the point of view of dark energy phenomenology, the Lagrangian (3) in such a regime is almost indistinguishable from a simple shift-symmetric k-essence model [31,32], which is formally recovered in the limit $\Lambda \rightarrow \Lambda_{2}$. We will argue, however, that the possible existence of higher-derivative operators below $\Lambda_{2}$ could nevertheless leave an observable imprint. Being irrelevant operators-in the renormalization group (RG)-flow sense- - their relative importance grows in the UV, i.e., at shorter distances. Exploiting the new observational window provided by gravitational astronomy, we will discuss in which cases the presence of such interactions can affect the gravitational dynamics at the length scales probed by black hole merger events and in particular during the ringdown phase.

As a consequence, in the following, we will be interested in EFTs that are able to describe, together with the evolution of the Universe at cosmological distances, at least black holes of the size probed by LIGO/Virgo, with a characteristic Schwarzschild radius of about $10 \mathrm{~km}$. We will therefore consider acceptable theories in which the scale of the UV completion can be as low as $\Lambda_{\mathrm{UV}} \sim$ $1 \mathrm{~km}^{-1}$. On the one hand, according to the condition coming from amplitudes' positivity (4), this requires that the scale suppressing the HD operators satisfies $\Lambda>\Lambda_{\min }^{\text {pos }} \sim$ $10^{5} \Lambda_{3}$. On the other hand, purely observational constraints can give a minimum allowed value $\Lambda_{\min }^{\text {obs }}$ for such a scale, which depending on the model can be either above or below $\Lambda_{\mathrm{min}}^{\mathrm{pos}}$. The greater of the two should be taken as the most stringent bound, i.e., $\Lambda>\max \left\{\Lambda_{\min }^{\text {pos }}, \Lambda_{\min }^{\text {obs }}\right\}$. See Fig. 1 for a schematic representation of the hierarchy of scales for $\Lambda$ discussed above and the various effects taking place at those scales.

\section{SHIFT-SYMMETRIC SCALAR-TENSOR THEORIES AND HAIRY BLACK HOLES}

A consistent way to include HD operators in the shiftsymmetric dark energy EFT at a scale $\Lambda$ that is much below the scale $\Lambda_{2}$ suppressing the operators with fewer derivatives is by doing so in the specific combinations that belong to the shift-symmetric (beyond) Horndeski class [16,33-35]. Indeed, such theories enjoy robust quantum properties due to their weakly broken Galileon invariance $[15,17]$. Their Lagrangian is

$$
S_{H+B H}=\int \mathrm{d}^{4} x \sqrt{-g} \sum_{i=2}^{5} \mathcal{L}_{i},
$$

where the $\mathcal{L}_{i}$ are functions of the metric $g_{\mu \nu}$ and the derivatives of the scalar field $\phi$. Specifically, we will write them as 


$$
\begin{aligned}
& \mathcal{L}_{2}=\Lambda_{2}^{4} G_{2}(X), \\
& \mathcal{L}_{3}=\frac{\Lambda_{2}^{4}}{\Lambda^{3}} G_{3}(X) \square \phi, \\
& \mathcal{L}_{4}=\frac{\Lambda_{2}^{8}}{\Lambda^{6}} G_{4}(X) R-2 \frac{\Lambda_{2}^{4}}{\Lambda^{6}} G_{4, X}(X)\left[(\square \phi)^{2}-\phi_{; \mu \nu} \phi^{; \mu \nu}\right] \\
& -\frac{F_{4}(X)}{\Lambda^{6}} \varepsilon^{\mu \nu \rho}{ }_{\sigma} \varepsilon^{\mu^{\prime} \nu^{\prime} \rho^{\prime} \sigma} \phi_{; \mu} \phi_{; \mu^{\prime}} \phi_{; \nu \nu^{\prime}} \phi_{; \rho \rho^{\prime}}, \\
& \mathcal{L}_{5}=\frac{\Lambda_{2}^{8}}{\Lambda^{9}} G_{5}(X) G_{\mu \nu} \phi^{; \mu \nu} \\
& +\frac{1}{3} \frac{\Lambda_{2}^{4}}{\Lambda^{9}} G_{5, X}(X)\left[(\square \phi)^{3}+2 \phi_{; \mu}{ }^{\nu} \phi_{; \nu}{ }^{\alpha} \phi_{; \alpha}{ }^{\mu}-3 \phi_{; \mu \nu} \phi^{; \mu \nu} \square \phi\right] \\
& -\frac{F_{5}(X)}{\Lambda^{9}} \varepsilon^{\mu \nu \rho \sigma} \varepsilon^{\mu^{\prime} \nu^{\prime} \rho^{\prime} \sigma^{\prime}} \phi_{; \mu} \phi_{; \mu^{\prime}} \phi_{; \nu \nu^{\prime}} \phi_{; \rho \rho^{\prime}} \phi_{; \sigma \sigma^{\prime}}
\end{aligned}
$$

where $X=g^{\mu \nu} \partial_{\mu} \phi \partial_{\nu} \phi / \Lambda_{2}^{4}$ is the scalar kinetic term and the semicolon denotes the covariant derivative. Note that not all the functions in $\mathcal{L}_{4}$ and $\mathcal{L}_{5}$ are independent, due to the requirement of satisfying the degeneracy conditions to ensure there are only 3 propagating degrees of freedom. Here, radiative corrections to the Galileon-breaking operators are suppressed by the ratio $\Lambda^{4} / \Lambda_{2}^{4}$.

In order to be able to probe the presence of the HD operators at the distance scales of black hole merger events, there must be substantial deviations of their gravitational dynamics from the prediction of General Relativity in the first place. A condition for this is that a sizable scalar field background, or hair, is sourced by the black holes themselves. This is not a generic feature of shift-symmetric scalar-tensor theories, though. Under some rather strong assumptions, namely staticity, spherical symmetry, asymptotic flatness, and regularity at the horizon, black hole solutions with a nontrivial scalar profile are severely restricted in such theories due to the existence of a no-hair theorem [36]. The assumption on asymptotic flatness is the first to go in the presence of a cosmological background, but any hair sourced by such background will have negligible effects on astrophysical black holes due to the great separation of the scales involved. Hair can also be generated by the time dependence of the inspiral and merger process. Nevertheless, this kind of hair will not persist in its later stages, i.e., the ringdown, where the black hole can be effectively described as a stationary background plus small perturbations that radiate away. Yet another possible source of hair is rotation, which will be generically present for a black hole produced in a merger process and may in contrast give rise to important effects. However, for slowly rotating black holes, rotation cannot source hair if there was none already in spherical symmetry [6], while for rapidly rotating black holes, this remains an open question. In any case, these types of hair do not provide a generic way to probe the presence of HD operators independently of the particular conditions of each event. ${ }^{5}$

As a starting point for this kind of analysis, in this paper, we will consider instead a kind of hair which is present even in the very symmetric ideal situation, sourced by the presence of the sGB operator [6],

$$
M_{\mathrm{P}} \alpha \phi \mathcal{R}_{\mathrm{GB}}^{2},
$$

where $\mathcal{R}_{\mathrm{GB}}^{2}$ is the Gauss-Bonnet invariant

$$
\mathcal{R}_{\mathrm{GB}}^{2}=R_{\mu \nu \rho \sigma} R^{\mu \nu \rho \sigma}-4 R_{\mu \nu} R^{\mu \nu}+R^{2} .
$$

We expect our estimates to also hold for slowly rotating black holes [38], while the general situation is beyond the scope of this work. The operator in (7) evades the no-hair theorem by breaking some of its assumptions in a more subtle way. Note also that it respects the shift symmetry nontrivially, due to the fact that $\mathcal{R}_{\mathrm{GB}}^{2}$ is a total derivative. Given that this operator leads to second-order equations of motion, it must be contained within the Horndeski part of (5) $\left(F_{4}=F_{5}=0\right)$. Indeed, it is equivalent to the choice

$$
G_{5} \propto \log (X)
$$

From the EFT standpoint, the allowed range for the sGB operator coupling $\alpha$ is huge. It is bounded from below by the size of its quantum corrections and from above by the requirement that the strong coupling scale is not below $\Lambda$. Namely,

$$
\frac{1}{M_{\mathrm{P}} \Lambda}<\alpha<\frac{M_{\mathrm{P}}}{\Lambda^{3}} .
$$

Hairy solutions in sGB theories have been studied mainly in the case when the only other operators present are the Einstein-Hilbert and the standard kinetic term for the scalar, $X$ [7-13]. In the language of the above Lagrangian, this case amounts to the choices $G_{2}=X, G_{4}=\Lambda^{6} / \Lambda_{3}^{6}$ and $G_{5}=-4 \frac{\Lambda^{9}}{\Lambda_{2}^{8}} M_{\mathrm{P}} \alpha \log (X)$, with the remaining functions set to zero. Black hole solutions in this context are known to have secondary hair, meaning there is no free parameter, or "charge," associated to them and regular solutions exist only if the coupling $\alpha$ is below a certain threshold [7]. It is also relevant to note that in this particular situation, there is no screening mechanism associated with scalar nonlinearities.

In our setup, on the contrary, other operators are present and can actually dominate over the standard kinetic term. Whether this happens or not depends on the size of the background quantities $X_{0}$ and $Z_{0}$, where $Z \equiv \nabla^{2} \phi / \Lambda^{3}$. Starting from asymptotically vanishing values, in the

\footnotetext{
${ }^{5}$ For a review about tests of black hole dynamics in modified theories of gravity, see e.g., Ref. [37].

${ }^{6}$ In Appendix A, we explain how to derive the upper bound
} on $\alpha$. 
presence of scalar hair, these quantities will grow as one approaches the vicinity of the black hole. However, their maximum values ultimately depend on the size of the sGB coupling $\alpha$. Since we are interested in probing the effect of higher-order operators, we will assume that $\alpha$ is large enough in order to be in a regime where $X_{0} \gg 1$ and $Z_{0} \gg 1$ at the Schwarzschild radius, and possibly farther away. Under this assumption, now suppose that the operator which dominates in this regime (besides the sGB one) has the following power counting:

$$
\Lambda_{2}^{4} G_{m+2}(X) Z^{m} \rightarrow \Lambda_{2}^{4} X^{n} Z^{m} .
$$

Here, $m$ always has to satisfy $m=0,1,2,3$, while $n$ is allowed to be any real number, since we are looking at the large- $X$ asymptotic behavior of the Lagrangian functions $G_{i}{ }^{7}$ Moreover, we also expect the deviation of the geometry from Schwarzschild, even close to the black hole, not to be very large if one has to be in agreement with current observations [13].

We now proceed in estimating the size of a background solution for the scalar hair, considering static and spherically symmetric configurations. Under the assumptions stated above, the scalar equation of motion schematically reads ${ }^{8}$

$$
\frac{\Lambda_{2}^{4}}{\Lambda^{3}} \partial^{2}\left(X_{0}^{n} Z_{0}^{m-1}\right) \sim M_{\mathrm{P}} \alpha \frac{r_{s}^{2}}{r^{6}},
$$

where on the right-hand side we are evaluating the GaussBonnet invariant $\mathcal{R}_{\mathrm{GB}}^{2}$ on a Schwarzschild background metric, with $r_{s}$ the Schwarzschild radius, which acts as the source for the scalar profile at leading order in $\alpha$. Assuming spherical symmetry and a power-law decay for the scalar hair $\phi_{0}(r)$ [i.e., $\phi_{0}(r) \sim c_{\phi} / r^{p}$, where $c_{\phi}$ and $p$ are constants], we can easily express the $Z_{0}$ on the background in terms of $X_{0}$ as

$$
Z_{0} \sim \frac{\Lambda_{2}^{2} X_{0}^{1 / 2}}{r \Lambda^{3}}
$$

and hence we are able to estimate $X_{0}$ to be

$$
X_{0}(r)^{n+(m-1) / 2} \sim M_{\mathrm{P}} \alpha \frac{r_{s}^{2}}{\Lambda_{2}^{2} r^{5}}\left(\frac{r \Lambda^{3}}{\Lambda_{2}^{2}}\right)^{m},
$$

where we demand that the exponent $\lambda=2 n+m-1>0$ in order for $X_{0}(r)$ to decay moving away from the source. Note

\footnotetext{
${ }^{7}$ Once the leading behavior (11) is chosen, this translates into an upper bound on the remaining $G_{i}$ functions in the large- $X$ limit, such that, on the solution, this assumption remains valid. Furthermore, quantum corrections will also generate for example $\Lambda^{4} Z^{p}$ terms, with $p$ a positive integer. Then, there is also the requirement that these will resum to a function $K(Z)$ which is small enough at large $Z$.

${ }^{8}$ Note that a contribution like $\partial\left(\partial \phi X^{n-1} Z^{m}\right)$ is also captured by the schematic form given.
}

that, after one includes extra operators besides a standard kinetic term, one can relax the theoretical bound on the size of the sGB operator coming from the regularity of solutions. See Appendix B for an example with the cubic Galileon.

\section{OBSERVABLE EFFECTS IN THE RINGDOWN}

One of the main goals of this paper is to show that, even if higher-derivative operators are negligible on cosmological scales, they can nevertheless become larger and possibly be tested at much shorter length scales. A promising opportunity to probe at least some of the self interactions of a scalar field and its coupling to gravity is provided by the observation of gravitational waves emitted during the merger of two black holes [39]. A robust signal of the presence of an additional degree of freedom can be imprinted on the waves emitted during the ringdown phase, when the newly formed and highly perturbed merger remnant relaxes to its equilibrium configuration. A potential deviation from the predictions of GR can have two origins: the scalar field may have a nontrivial background that deforms the geometry of the final black hole, or there can be a mixing between gravitational and scalar perturbations around the background solution [37]. Both will ultimately affect the spectrum of the quasinormal modes (QNM). In the following, we will estimate these two effects for a black hole formed in a merger, at a typical distance of the order of the light ring, $r \sim r_{s}$, where their contribution to the QNM spectrum is the largest. We stress that this will just be a rough estimate of the order of magnitude of these effects. A full computation, though very important and eventually necessary, is beyond the scope of this paper. In fact, these effects have been carefully studied in the particular case of sGB plus canonical kinetic term in e.g., Refs. [13,40].

\section{A. Background geometry}

The simplest way to estimate how the presence of a scalar background modifies the spacetime geometry around the black hole, with respect to the Schwarzschild metric, is to compare the sGB operator (7), evaluated on the unperturbed metric and using the solution (14) for the scalar, with $M_{\mathrm{P}}{ }^{2}$ times the black hole curvature $\mathcal{R} \sim r_{s} / r^{3}$ (see Appendix $\mathrm{C}$ ), as functions of the distance $r$. The ratio between these two quantities will be

$$
\varepsilon_{0}(r) \equiv \frac{M_{\mathrm{P}} \alpha \phi_{0} \mathcal{R}_{\mathrm{GB}}^{2}}{M_{\mathrm{P}}^{2} \mathcal{R}} \sim \alpha \frac{r_{s} \Lambda_{2}^{2}}{M_{\mathrm{P}} r^{2}} \sqrt{X_{0}(r)} .
$$

Deviations from the Schwarzschild geometry of $\mathcal{O}(1)$ are possible in principle in light of the above expression.

\section{B. Mixing}

The second source of modification for the QNM spectrum is due to the appearance of mixing terms between scalar and gravitational modes in the quadratic Lagrangian 
expanded around the spherically symmetric background solution. Such terms can be present even if the metric is very close, or exactly equal as in the case of the so-called stealth solutions, to Schwarzschild [4,41].

The sGB operator induces a kinetic mixing which schematically has the following form,

$$
M_{\mathrm{P}} \alpha \phi \mathcal{R}_{\mathrm{GB}}^{2} \supset \alpha \frac{r_{s}}{r^{3}} \partial h_{c} \partial \pi \equiv \mathcal{Z}_{\mathrm{mix}}^{\mathrm{GB}} \partial h_{c} \partial \pi,
$$

where $h_{c}$ stands for a canonically normalized metric perturbation and $\pi \equiv \phi-\phi_{0}(r)$. All the other HD operators that appear in (6) also give rise to a mixing, and in fact one can easily check (see Appendix C) that the contribution from the dominating operator (11) - for a regular function $G_{i}(X)$-goes as

$$
\mathcal{Z}_{\text {mix }}^{H} \sim \frac{r_{s}}{r} \mathcal{Z}_{\text {mix }}^{\mathrm{GB}},
$$

on solutions of the equation of motion (12). Therefore, at $r \sim r_{s}$, both contributions to the kinetic mixing are of the same order, but with inequivalent contractions due to their different structure. Presumably, their impact on the QNM spectrum will differ. This will be studied elsewhere.

To estimate the impact of such a mixing on the ringdown, one has to compare its size to the diagonal elements of the kinetic matrix. As discussed in the previous section, this is where a big difference with respect to most of the literature about Gauss-Bonnet hair appears. If in addition to the sGB operator (7) only $(\partial \phi)^{2}$ is present in the scalar Lagrangian, the kinetic term for the perturbation $\pi$ around the background receives no other contributions, and it is therefore canonically normalized. The coefficient $\mathcal{Z}_{\text {mix }}^{\mathrm{GB}}$ in (16) then gives the typical size of the effect. In the class of theories considered in this paper, on the other hand, additional operators must be present. Even in the minimal setup, the $G_{2}(X)$-type operators must be added, because they have to provide the stress-energy tensor responsible for the accelerated expansion of the Universe, together with all the interactions generated by quantum corrections, as required by a consistent EFT description. In this case, the kinetic term for scalar perturbations will be provided with $r$-dependent contributions that grow getting closer to the black hole, $\mathcal{Z}_{\pi}(r)(\partial \pi)^{2}$. As we discussed in the previous section when solving the equation of motion, if a value of $r$ is reached such that the dimensionless quantities $Z_{0}$ and $X_{0}$ evaluated on the background are much larger than 1 , one can identify the contribution that dominates in this regime and, in this case, estimate the leading correction to the kinetic term, which is given by

$$
\Delta \mathcal{Z}_{\pi} \sim X_{0}^{n-1} Z_{0}^{m} \sim M_{\mathrm{P}} \alpha \frac{r_{s}^{2}}{r^{5} \Lambda_{2}^{2}} \frac{1}{\sqrt{X_{0}(r)}} .
$$

When $\mathcal{Z}_{\pi} \gg 1$, the physical effect of the mixing is obtained only after the scalar perturbation is canonically normalized $\pi=\pi_{c} / \sqrt{\mathcal{Z}_{\pi}}$, and the result reads

$$
\varepsilon_{\text {mix }}(r) \equiv \frac{\mathcal{Z}_{\text {mix }}^{\mathrm{GB}}}{\sqrt{\mathcal{Z}_{\pi}}} \sim \sqrt{\alpha}\left(\frac{\Lambda_{2}^{2}}{M_{\mathrm{P}} r}\right)^{1 / 2} X_{0}(r)^{1 / 4} .
$$

The same phenomenon, the existence of a large scalar background and, as a consequence, of large corrections to the coefficient of the field perturbation close to massive sources appears in so-called screening mechanisms. ${ }^{9}$ In those cases, the field redefinition, which is necessary to canonically normalize the scalar perturbation, produces a suppression of the direct coupling of $\pi$ to matter. The fifthforce exchange of the scalar is thus reduced. For fixed $r_{s}$, the $r$-dependence of (19) and (14) indicates that the kinetic mixing effect is maximum at close range to the black hole, i.e., $r \sim r_{s}$. Moreover, this effect is stronger for smaller black holes,

$$
\varepsilon_{\text {mix }}\left(r \sim r_{s}\right) \propto r_{s}^{-\frac{(n+1)}{2 n+m-1}} .
$$

A measurement of this effect for various black holes of different masses would allow us to constrain the form of the dominant operator ( $n$ and $m$ ) through the above dependence.

For later use, let us consider two different systems of Schwarzschild radii $r_{s 1}$ and $r_{s 2}$, respectively. The ratio of the mixing effects at distances $r_{1}$ and $r_{2}$ of each source is

$$
\frac{\varepsilon_{\text {mix }}\left(r_{1}, r_{s 1}\right)}{\varepsilon_{\text {mix }}\left(r_{2}, r_{s 2}\right)} \sim\left(\frac{r_{2}}{r_{1}}\right)^{1 / 2}\left[\left(\frac{r_{2}}{r_{1}}\right)^{5-m} \frac{r_{s 1}^{2}}{r_{s 2}^{2}}\right]^{\frac{1}{4 n+2(m-1)}},
$$

where we used Eqs. (19) and (14). Notice that the way this effect scales with distances and masses of the systems is only dependent on the choice of the dominant operator (i.e., on $n$ and $m$ ). Other parameters such as the sGB coupling $\alpha$ and the scale $\Lambda$ drop from the above expression.

Another interesting remark is the fact that the sizes of both the effect on the background geometry $\varepsilon_{0}$ and the effect from kinetic mixing $\varepsilon_{\text {mix }}$ are not independent. Indeed, notice that in general one has

$$
\varepsilon_{0}(r)=\frac{r_{s}}{r} \varepsilon_{\text {mix }}(r)^{2},
$$

and, therefore, the kinetic mixing effect will always dominate over the effect on the background geometry if both are to be at most of $\mathcal{O}(1)$ at the light ring.

\section{CONSTRAINTS FROM TESTS OF GRAVITY}

The absolute strength of the effects around black holes discussed above depends on both the choice of coupling $\alpha$ as well as on the form ( $n$ and $m$ ) and the scale $\Lambda$ of the other operators that are present in the Lagrangian. However, the presence of a scalar background may also introduce effects at different scales, where current observations put strong bounds to deviations from GR. Already in LIGO/Virgo

\footnotetext{
${ }^{9}$ For a review, see e.g., Refs. [42,43].
} 
events, the absence of an observed dephasing of the gravitational wave signal from the one predicted by GR puts an upper bound on the strength of scalar wave emission $[44,45]$. In a different regime, there are also very precise tests of gravitational physics in the Solar System. One of the strongest bounds of this type comes from Lunar Laser Ranging measurements that put strict constraints on the existence of any kind of fifth force at about the $10^{-10}$ level at distances of the Earth-Moon orbit $[46,47]$. These kinds of bounds will limit the choice of $\alpha, \Lambda$ and of the allowed operators. A given choice of $\Lambda$ will furthermore impact the strength of Vainshtein screening and the size of the Vainshtein radii for various systems. It is therefore advisable to revisit situations where this kind of mechanism is necessary in order to agree with observations. We will now discuss these constraints in more detail.

\section{A. Direct scalar-matter coupling}

When matter is present, it is important to know which is the dominant source for the scalar background. Indeed, besides the sGB operator discussed so far, in general we can expect the scalar to be directly coupled to matter, which can source a scalar profile around matter sources but does not affect the solution around black holes. The sGB operator, instead, sources the scalar in both situations. Let us parametrize the size of such a direct scalar-matter coupling relative to the strength of gravity by $\delta$,

$$
\frac{\delta}{M_{\mathrm{P}}} \phi T \text {. }
$$

Considering a kinetic mixing of cosmological origin, we expect at least that $\delta>\Lambda_{3}^{3} / \Lambda^{3}$ (Appendix D). Due to this direct coupling, a matter source of mass $M_{*}$ will generate a scalar background with an associated Vainshtein radius of order

$$
\tilde{r}_{v} \equiv \frac{1}{\Lambda}\left(\delta \frac{M_{*}}{M_{\mathrm{P}}}\right)^{1 / 3}=\frac{\Lambda_{3}}{\Lambda} \delta^{1 / 3} r_{v}
$$

where $r_{v}$ is the usually quoted Vainshtein radius (as sourced by nonlinear interactions suppressed by $\Lambda_{3}$ in the presence of a $\phi T / M_{\mathrm{P}}$ scalar-matter coupling) [43]. The intensity of the screening effect is instead given by the size of $\sqrt{\mathcal{Z}_{\pi}}$, which grows quickly once inside $\tilde{r}_{v}$, but it is $\mathcal{O}(1)$ farther away from the source. According to Eq. (24), there is in general a much smaller Vainshtein radius compared to the standard case, i.e., $\tilde{r}_{v} \ll r_{v}$, and one should check that this does not enter in conflict with current tests of gravity at various scales. Indeed, if screening is needed in order to avoid fifth force constraints, once $\tilde{r}_{v}$ becomes the size of the system being considered or smaller, one might run into trouble. The way around is to bring $\delta$ down, which, although it further decreases $\tilde{r}_{v}$, also alleviates the problem that screening is trying to solve in the first place.
Consider the smallest value of $\Lambda$ that is generically consistent with $c_{T}=1$, i.e., $\Lambda \sim 10^{3} \Lambda_{3} \sim 1 \mathrm{~km}^{-1}$. With this choice and a direct coupling of gravitational strength, $\delta \sim 1$, large systems such as galaxies or galaxy clusters would be in the situation described above, where the fifth force becomes unscreened in their outer regions. This can potentially lead to some tension, and suggests that $\delta \ll 1$. However, if not of gravitational strength, there is no other well-motivated value for the coupling $\delta$ other than the one generated by kinetic mixing of cosmological origin. With this value of $\Lambda$, we have that $\delta \sim 10^{-9}$ (see Appendix D), so we will assume that $\delta$ is approximately of this size.

Now, let us consider the ratio between the source terms of the scalar background for the Earth-Moon system, again assuming that the deviation of the geometry from GR is not larger than $\mathcal{O}(1)$ (and therefore $T \sim M_{\mathrm{P}}{ }^{2} \mathcal{R}$ ),

$$
\frac{M_{\mathrm{P}} \alpha \mathcal{R}_{\mathrm{GB}}^{2}}{\delta M_{\mathrm{P}} \mathcal{R}} \sim \frac{\alpha}{\delta} \frac{r_{s}^{\oplus}}{r_{E-M}^{3}},
$$

where $r_{s}^{\oplus}$ is the Schwarzschild radius of the Earth and $r_{E-M}$ is the typical radius of the orbit of the Moon around the Earth. If this ratio is equal to or larger than 1, the background is sourced by sGB also at this scale. In particular, both the estimations for the scalar background and the size of the mixing are then given by the same expressions as for black holes, Eqs. (14) and (19), respectively, appropriately substituting $r_{s}$ by $r_{s}^{\oplus}$. We will assume this is the case, and we will later check that this is indeed satisfied for specific choices of $\alpha, \Lambda$ and the form of the leading HD operator ( $n$ and $m$ ).

\section{B. Scalar wave emission in the inspiral phase}

An important bound comes directly from the effect that a scalar wave emission can have on the inspiral phase of a binary black hole merger. The current best bound on the effective sGB coupling [13] at the scales probed during the inspiral, i.e., at $r_{\text {insp }}$, comes from the GW151226 event, due to the large number of observed cycles during this phase [44]. In terms of the effective value of the coupling $\alpha_{\text {insp }}$ seen by scalar perturbations during this phase, the bound reads ${ }^{10}$

\footnotetext{
${ }^{10}$ Note that the bound from Ref. [13] is obtained using a full simulation of inspiral, merger, and ringdown phases, which is stronger by an order of magnitude than the corresponding pure inspiral constraints $[48,49]$. In an abuse of notation, we will nevertheless label the correspondingly constraint coupling $\alpha_{\text {insp }}$ and analogously for related parameters. The presence of HD operators in addition to the sGB interaction will likely affect particularly the highly nonlinear merger phase, potentially altering the value of the bound on $\alpha_{\text {insp. Since we are interested in }}$ approximate order of magnitude estimates here, we will leave a refinement of our analysis taking into account these effects in more detail for future work and assume $\alpha_{\text {insp }}$ can approximately be bounded as discussed above.
} 


$$
\alpha_{\text {insp }} \equiv \frac{\alpha}{\sqrt{\mathcal{Z}_{\pi}\left(r_{\mathrm{insp}}\right)}}<(2.7 \mathrm{~km})^{2}
$$

where the denominator accounts for the effect of the Vainshtein screening. Again, this is an important difference with most works that studied observational bounds on the sGB coupling, where usually this effect is not present due to the absence of operators which modify $\mathcal{Z}_{\pi}$. Nevertheless, here we are only naively estimating how screening will affect the observable coupling, since in dynamical situations such as during a merger it is not yet clear how effective this mechanism is [50,51]. Using Eqs. (16), (19), and (26), we can then obtain a bound on $\varepsilon_{\text {mix }}$ (we explicitly evaluate these bounds in Sec. VIC), where

$$
\varepsilon_{\text {mix }}(\text { insp })=\left.\alpha_{\text {insp }} \frac{r_{s}}{r^{3}}\right|_{\text {insp }} .
$$

This can in turn be related to the mixing at the light ring $\left(r \sim r_{s}\right)$ of a different black hole with Schwarzschild radius $r_{s}$ by Eq. (21),

$\frac{\varepsilon_{\text {mix }}\left(r_{s}\right)}{\varepsilon_{\text {mix }}(\text { insp })} \sim\left(\frac{r_{\text {insp }}}{r_{s}}\right)^{1 / 2}\left[\left(\frac{r_{\text {insp }}}{r_{s}}\right)^{5-m} \frac{r_{s}^{2}}{\left(r_{s}^{\text {insp }}\right)^{2}}\right]^{\frac{1}{4 n+2(m-1)}}$.

The bound (26) then implies a bound on this quantity as well.

\section{Solar System tests}

Finally, we now consider constraints coming from highly precise tests of gravity in the Solar System. As discussed in the previous section, we expect effects from kinetic mixing to dominate observable deviations from GR around black holes. However, in order to avoid violating fifth force constraints, we must check that the same kind of effect is negligible in the Solar System. In particular, at the scale of the Earth-Moon orbit, the mixing must stay below the $10^{-10}$ level, in order to satisfy the Lunar Laser Ranging constraints $[46,47]$. In other words,

$$
\varepsilon_{\text {mix }}(E-M) \lesssim 10^{-10} .
$$

Assuming the same operator is dominating the kinetic term in both scenarios (same $m$ and $n$ ), the ratio (between inspiral and Solar System $\varepsilon_{\text {mix }}$ ) will be independent of the coupling $\alpha$ and the scale $\Lambda$. Again, using Eq. (21),

$\frac{\varepsilon_{\text {mix }}(E-M)}{\varepsilon_{\text {mix }}(\text { insp })} \sim \sqrt{\frac{r_{\text {insp }}}{r_{E-M}}}\left[\left(\frac{r_{\text {insp }}}{r_{E-M}}\right)^{5-m}\left(\frac{r_{s}^{\oplus}}{r_{s}^{\text {insp }}}\right)^{2}\right]^{\frac{1}{4 n+2(m-1)}}$.

While (26) and (27) then bound $\varepsilon_{\text {mix }}$ on inspiral scales (as explicitly discussed for GW151226 above), relating this bound to the Solar System constraint (29) via (30) then allows us to restrict $n$ and $m$, i.e., to restrict the form of the leading effective HD operator (11).

\section{VIABLE MODELS}

Now that we have discussed both the observable signatures and constraints, in this section, we proceed to identify explicit models that are viable, i.e., consistent with the above constraints. In what follows, we take the approach to first and foremost maximize the possible observational effects, and then to see which of these models can satisfy the various constraints. Therefore, we will assume that the bound (26) is saturated. With this, we maximize both the size of the kinetic mixing at the light ring of black holes, Eq. (28), as well as its size around the Earth-Moon system, Eq. (30).

\section{A. Condition on the allowed operators}

Having the maximum effect around the Earth-Moon system gives a conservative condition on the allowed operators, i.e., $n$ and $m$. Indeed, we must demand that the right-hand side of Eq. (30) satisfies the Solar System bound (29). This condition implies that in practice $n$ cannot be arbitrarily large, for a given $m$; otherwise, the scaling of the effect in going from the binary black hole inspiral down to the Earth-Moon would be too mild to accommodate this bound. The excluded region in the $n-m$ plane is shown in Fig. 2, shaded in blue. As also seen in Table I, some

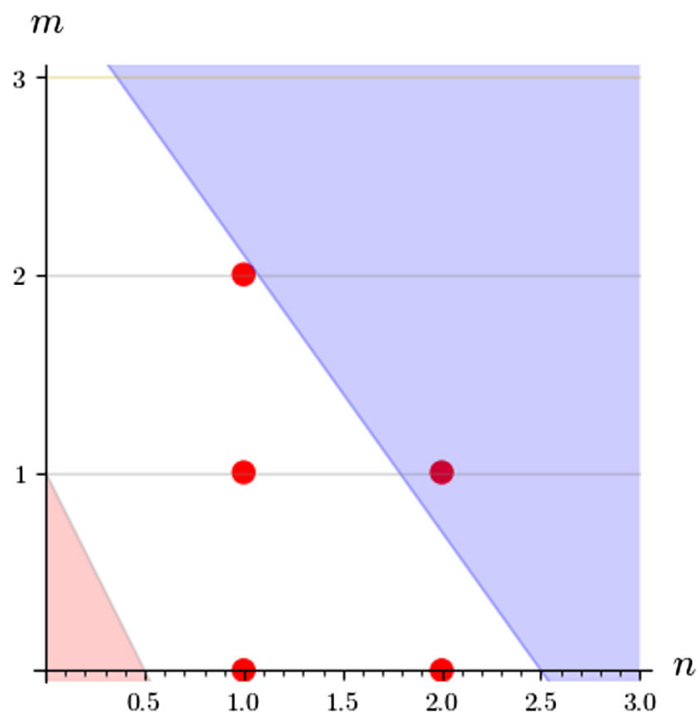

FIG. 2. Allowed values of $n$ for a given $m=0,1,2,3$, assuming the inspiral bound (26) saturates. The plot is cut off toward the lower left, since $\lambda=2 n+m-1$ goes negative in the red region and solutions for $X_{0}(r)$ no longer decay moving away from the source. Instead, the cut on the top right indicates the Lunar Laser Ranging bound (29) is violated. Note that this analysis does not exclude $(\square \pi)^{m}$ operators with $m=2,3$, but such operators of course are either total derivatives or ghostly. Viable operators that remain are e.g., $X, X^{2}, X Z$, and $X Z^{2}$, with $X^{2} Z$ also being borderline acceptable. These are denoted by the red dots. 
TABLE I. Examples of allowed operators, shown as red dots in Fig. 2, and the size of their expected kinetic mixing effect both near a black hole with $r_{s}=10 \mathrm{~km}$ and around the Earth-Moon when assuming the inspiral bound (26) is saturated. We also show $\Lambda_{\min }^{\mathrm{obs}}$ and the corresponding $\alpha$ satisfying the various observational bounds discussed.

\begin{tabular}{cccccccc}
\hline \hline$m$ & $n$ & Operator & $\varepsilon_{\text {mix }}\left(r \sim r_{s}\right)$ & $\varepsilon_{\text {mix }}(E-M)$ & $\Lambda_{\min }^{\text {obs }}\left[\mathrm{km}^{-1}\right]$ & $\Lambda_{\min }^{\text {pos }}\left[\mathrm{km}^{-1}\right]$ & $\alpha\left[\mathrm{km}^{2}\right]$ \\
\hline 0 & 1 & $X$ & $10^{-1}$ & $3 \times 10^{-21}$ & $3 \times 10^{3}$ & $10^{2}$ & 10 \\
0 & 2 & $X^{2}$ & $10^{-3}$ & $10^{-11}$ & 10 & $10^{2}$ & $10^{9}$ \\
1 & 1 & $X Z$ & $10^{-3}$ & $10^{-13}$ & 10 & $10^{2}$ & $3 \times 10^{9}$ \\
1 & 2 & $X^{2} Z$ & $3 \times 10^{-4}$ & $3 \times 10^{-10}$ & 1 & $10^{2}$ & $3 \times 10^{12}$ \\
2 & 1 & $X Z^{2}$ & $3 \times 10^{-4}$ & $10^{-10}$ & 1 & $10^{2}$ & $3 \times 10^{14}$ \\
\hline \hline
\end{tabular}

well-known cases such as the cubic or quartic Galileon [14] are permitted.

\section{B. sGB coupling $\alpha$ and HD operator scale $\Lambda$}

As already mentioned, the choice of saturating the inspiral bound (26) sets the size of the kinetic mixing effect at the Schwarzschild radius. This gives a relation between $\alpha$ and $\Lambda$ for a given choice of operator ( $n$ and $m$ ). Indeed, by using Eqs. (19) and (14), we find that

$$
\alpha^{2 n+m} \Lambda^{3 m} \sim \frac{M_{\mathrm{P}}^{2 n+m-2}}{\Lambda_{2}^{4(n-1)}} \frac{r_{\text {insp }}^{2 n+4}}{r_{s, \text { insp }}^{2}} \varepsilon_{\text {mix }}(\text { insp })^{4 n+2 m-2} .
$$

Notice that in the particular case of $m=0$, this fixes $\alpha$ directly, since $\Lambda$ plays no role in that case.

There is still the freedom to choose the scale $\Lambda$, as long as it is above $10^{5} \Lambda_{3} \sim 10^{2} \mathrm{~km}^{-1}$ in order to satisfy the requirement from amplitudes, Eq. (4), but still well below $\Lambda_{2}$. We also recall that, as discussed in Sec. VA, we are working under the assumption that the dominant source for the scalar profile in the Solar System is the sGB operator, rather than the direct coupling of cosmological origin, parametrized by $\delta$. So, from (25), a further condition on $\alpha$ and $\Lambda$ is that

$$
\alpha>\delta \frac{r_{E-M}^{3}}{r_{s}^{\oplus}} \sim\left(\frac{\Lambda_{3}}{\Lambda}\right)^{3} \frac{r_{E-M}^{3}}{r_{s}^{\oplus}}
$$

This can be plugged back into (31) and solved for a lower bound on $\Lambda$. In most cases, this will be a weaker bound than the one from amplitudes' positivity, but not always-see Table I. We call the stronger of these two lower bounds $\Lambda_{\text {min }}(n, m)$, the minimum value of $\Lambda$ one can consistently choose for a given operator, and separately refer to $\Lambda_{\min }^{\text {obs }}$ and $\Lambda_{\text {min }}^{\text {pos }}$ for the bounds from observations (i.e., from inspiral, background sourcing, Lunar Laser Ranging, and speed of gravitational wave constraints) and from positivity requirements.

\section{Examples}

Let us now look for explicit examples of models that satisfy all the conditions that were discussed in the previous sections. We take for the radius of the Earth-Moon orbit $r_{E-M} \sim 3 \times 10^{5} \mathrm{~km}$ and the Schwarzschild radius of the Earth $r_{s}^{\oplus} \sim 10^{-5} \mathrm{~km}$, while we use the GW151226 values for the inspiral quantities, ${ }^{11} r_{s}{ }^{\text {insp }} \sim 30 \mathrm{~km}$ and $r_{\text {insp }} \sim 300 \mathrm{~km}$. This immediately allows us to evaluate the condition on $n$ and $m$ which accounts for the Lunar Laser Ranging bound (29). A complementary condition on these exponents was already mentioned after Eq. (14), namely the requirement that $2 n+m-1>0$, related to our demand that the background solution $X_{0}(r)$ decays moving away from the source. We plot these two conditions together in Fig. 2, where the shaded regions are excluded. Examples of viable theories are marked as red dots within the allowed region.

For these examples, we show in Table I the size of the kinetic mixing effect close to a black hole of size $r_{s} \sim 10 \mathrm{~km}$, as well as its size around the Earth-Moon system. Recall that, by saturating the bound (26), the quoted values for $\varepsilon_{\text {mix }}$ are upper bounds for each model. We also quote $\Lambda_{\mathrm{min}}^{\mathrm{obs}}$, i.e., the smallest value of $\Lambda$ compatible with observations, including $c_{T}=1$, and the corresponding $\alpha$ according to Eq. (31).

Notice that for all the models shown in Table I, the kinetic mixing effect is always much smaller than 1 . According to Eq. (22), the deviation of the background geometry from Schwarzschild is then even smaller. A question one might ask is whether it is possible to make both these effects to be $\mathcal{O}(1)$ at $r \sim r_{s}$. While the inspiral bound puts a tight constraint on this possibility, this can nevertheless still be achieved if the kinetic mixing scales steeply enough as one approaches the Schwarzschild radius. From Eq. (21), one can readily see that, fixing all the scales but $r_{1}=r$, this effect scales as

$$
\varepsilon_{\text {mix }}(r) \propto r^{-\frac{(n+2)}{2 n+m-1}} .
$$

This dependence is stronger as $n$ decreases, meaning that models with "maximal" $\varepsilon_{\text {mix }}\left(r \sim r_{s}\right)$ would fall on the

\footnotetext{
${ }^{11}$ Actually, the inspiral phase spans a range of distances $r_{\text {insp }}$ between roughly 3 and 30 times the Schwarzschild radius of the black holes, $r_{s}^{\text {insp }}$ [49]. We take an intermediate value which should be acceptable for our purpose of giving order of magnitude estimates for the effects at the light ring.
} 
leftmost part of the plot in Fig. 2, especially in the region with $n<1{ }^{12}$ Such exotic models can nevertheless be considered acceptable from the EFT point of view, if one intends to remain agnostic about the UV completion of the theory. Indeed, as discussed around Eq. (11), in the regime for which $X_{0} \gg 1$, there is not necessarily a single operator with an integer value of $n$ that dominates, but rather an infinite tower of operators which collectively show an asymptotic behavior for large $X$ that is compatible with a non integer $n$. This means that $\mathcal{O}(1)$ mixing as well as $\mathcal{O}(1)$ deviations from a Schwarzschild background can be achieved with a judicious choice of HD operators in addition to the sGB one, while remaining consistent with all other constraints discussed here.

\section{CONCLUSIONS}

In this work, we have explored the possibility that the dynamics of a scalar field $\phi$ responsible for the accelerated expansion of the Universe can be probed in the strong gravity regime of a black hole coalescence. Our analysis relies on three assumptions: i) the interactions of $\phi$ are shift symmetric; ii) a scalar hair is generated around the final black hole, sourced by a linear coupling between the field and the Gauss-Bonnet operator; and iii) the spin of the black hole is ignored.

Within these conditions, we have found that the presence of certain scalar self-interactions can affect, in an observable way, the spectrum of quasinormal modes emitted during the black hole ringdown. This conclusion is robust, at least from an EFT perspective. The dynamics of the new degree of freedom is parametrized in a general way, based on exact and approximate symmetries which provide welldefined power-counting rules for the derivative and field expansions within the effective Lagrangian. The regime of applicability of the EFT is also imposed to be consistent with the strongest constraints coming from amplitudes' positivity conditions derived up to now in this class of theories [27], which also ensures compatibility with constraints on the speed of gravitational waves [18].

The details of the resulting deviations from GR predictions are, on the other hand, model dependent, even if they are all ultimately originated by the presence of the sGB coupling. This is because at scales of order of the light ring the leading effect, depending on the details of the scalar theory, can be given by different operators. While in the paper we provide only an order of magnitude estimate of such effects, a more complete computation would be useful: the explicit results for QNM spectra obtained so far in the literature are insufficient to fully characterize the potential experimental signatures of this scenario. They are in fact obtained in the limiting case where the only other

\footnotetext{
${ }^{12}$ Indeed, this trend is also visible in Table I, where it is clear that $\varepsilon_{\text {mix }}\left(r \sim r_{s}\right)$ increases with decreasing $n$.
}

operator present in the scalar Lagrangian-a part from Gauss-Bonnet-is the kinetic term.

The variety of possible sources of new effects in the gravitational waveform emitted during the ringdown suggests that, instead of studying each and every case separately, it would be useful to adopt a more model independent approach, like the one recently proposed in [52], to compute the QNM spectrum.

Note that the observable effects discussed here are at the $0.03-0.1$ percent level. While $\mathcal{O}(1)$ deviations from Schwarzschild background solutions are already strongly constrained, the sensitivity of current experiments will likely not be enough to probe effects of this size. However, we stress that deviations from GR observable with the next generation of detectors [53] are well motivated, since their presence is quite generic and robust.

Finally, we wish to reiterate that the results presented here mean that, in the presence of "hair," the nature of dark energy can be probed with strong gravity observables. While several orders of magnitude separate the scales associated to these regimes, we have shown that a welldefined set of theories is predictive over this range of scales and yields observable signatures in binary black hole systems.

\section{ACKNOWLEDGMENTS}

We acknowledge comments from and useful discussions with P. Creminelli, P. Ferreira, L. Hui, S. Melville, P. Pani, R. Penco, F. Serra, and K. Yagi. J. N. acknowledges support from Dr. Max Rössler, the Walter Haefner Foundation, and the ETH Zurich Foundation. L. S. is supported by Simons Foundation Award No. 555117. E. T. and L. G. T. are supported in part by the MIUR under Contracts No. 2015P5SBHT and No. 2017FMJFMW.

\section{APPENDIX A: NONRENORMALIZATION OF GAUSS-BONNET AND WEAKLY BROKEN GALILEON SYMMETRY}

The upper bound (10) on the coupling $\alpha$ of the sGB operator (7) can be obtained by requiring that the strong coupling scale of the theory is not below $\Lambda$. A simple way to derive it is by comparing loop diagrams involving the sGB term with tree-level operators in the EFT (3). However, in order to get the correct result, one should note that in any quantum loop involving the sGB operator, at the leading order in $1 / M_{\mathrm{P}}$, any scalar leg attached to sGB vertices comes always with at least two derivatives. In other words, the sGB operator satisfies the power counting of the class of operators with weakly broken Galileon symmetry, introduced in Refs. [15,17]. ${ }^{13}$ In the following, we provide

\footnotetext{
${ }^{13}$ This is in agreement with the fact that the sGB combination is equivalent to a quintic Horndeski operator, provided a very specific choice of the Horndeski function $G_{5} \propto \log (X)$ [54].
} 
an explicit check of the latter statement, which in turn will imply that $\alpha$ is bounded from above by $M_{\mathrm{P}} / \Lambda^{3}$.

Potentially dangerous contributions, inducing potentially large quantum corrections to the couplings of the operators of the form $(\partial \phi)^{2 n}$, are those coming from sGB vertices with two graviton lines, which carry the least suppression in powers of $1 / M_{\mathrm{P}}$. We will check that, after tedious integrations by parts, those vertices can in general be rewritten in such a way that the scalar field always carries at least two derivatives,

$$
M_{\mathrm{P}} \alpha \phi \mathcal{R}_{\mathrm{GB}}^{2} \supseteq \frac{\alpha}{M_{\mathrm{P}}} \phi\left(\partial^{2} h_{c}\right)^{2} \sim \frac{\alpha}{M_{\mathrm{P}}} h_{c} \partial^{2} \phi \partial^{2} h_{c},
$$

implying therefore that they actually do not renormalize $(\partial \phi)^{2 n}$. To this end, we expand the Riemann tensor, the
Ricci tensor, and the curvature scalar at linear order in the metric perturbation,

$$
\begin{aligned}
& R_{\mu \nu \rho \sigma}= \frac{1}{2}\left(\partial_{\rho} \partial_{\nu} h_{\mu \sigma}+\partial_{\sigma} \partial_{\mu} h_{\nu \rho}-\partial_{\sigma} \partial_{\nu} h_{\mu \rho}-\partial_{\rho} \partial_{\mu} h_{\nu \sigma}\right) \\
&+\mathcal{O}\left(h^{2}\right), \\
& R_{\mu \nu}=\frac{1}{2}\left(\partial_{\sigma} \partial_{\nu} h_{\mu}^{\sigma}+\partial_{\sigma} \partial_{\mu} h_{\nu}^{\sigma}-\partial_{\mu} \partial_{\nu} h-\partial_{\sigma} \partial^{\sigma} h\right)+\mathcal{O}\left(h^{2}\right), \\
& R=\partial_{\mu} \partial_{\nu} h^{\mu \nu}-\partial_{\sigma} \partial^{\sigma} h+\mathcal{O}\left(h^{2}\right) .
\end{aligned}
$$

Plugging this into the definition of the Gauss-Bonnet operator, we obtain

$$
\begin{aligned}
\phi \mathcal{R}_{\mathrm{GB}}^{2}= & \phi\left[\partial_{\mu} \partial_{\nu} h_{\rho \sigma} \partial^{\mu} \partial^{\nu} h^{\rho \sigma}+\partial_{\mu} \partial_{\nu} h_{\rho \sigma} \partial^{\rho} \partial^{\sigma} h^{\mu \nu}-2 \partial_{\mu} \partial_{\nu} h_{\rho \sigma} \partial^{\mu} \partial^{\rho} h^{\nu \sigma}-2 \partial_{\sigma} \partial_{\nu} h_{\mu}^{\sigma} \partial_{\rho} \partial^{\nu} h^{\rho \mu}-2 \partial_{\sigma} \partial_{\nu} h_{\mu}^{\sigma} \partial_{\rho} \partial^{\mu} h^{\rho \nu}\right. \\
& +4 \partial_{\mu} \partial_{\nu} h \partial^{\sigma} \partial^{\mu} h_{\sigma}^{\nu}+4 \square h_{\mu \nu} \partial_{\sigma} \partial^{\mu} h^{\sigma \nu}-\partial_{\mu} \partial_{\nu} h \partial^{\mu} \partial^{\nu} h-2 \partial_{\mu} \partial_{\nu} h \square h^{\mu \nu}-\square h_{\mu \nu} \square h^{\mu \nu}+\partial_{\mu} \partial_{\nu} h^{\mu \nu} \partial_{\rho} \partial_{\sigma} h^{\rho \sigma} \\
& \left.-2 \partial_{\mu} \partial_{\nu} h^{\mu \nu} \square h+(\square h)^{2}\right] .
\end{aligned}
$$

Finally, after straightforward integrating by parts, we find

$$
\begin{aligned}
\phi \mathcal{R}_{\mathrm{GB}}^{2}= & \square \phi \partial_{\nu} h_{\rho \sigma} \partial^{\nu} h^{\rho \sigma}-\partial_{\mu} \partial_{\nu} \phi \partial^{\nu} h_{\rho \sigma} \partial^{\mu} h^{\rho \sigma}-\partial_{\rho} \partial_{\nu} \phi \partial_{\sigma} h^{\sigma \mu} \partial_{\mu} h^{\nu \rho}+\partial_{\rho} \partial_{\sigma} \phi \partial_{\nu} h^{\sigma \mu} \partial_{\mu} h^{\rho \nu}+\partial_{\mu} \partial_{\nu} \phi \partial_{\sigma} h^{\sigma \mu} \partial_{\rho} h^{\rho \nu} \\
& -\partial_{\mu} \partial_{\sigma} \phi \partial_{\nu} h^{\sigma \mu} \partial_{\rho} h^{\rho \nu}-2 \partial_{\mu} \partial^{\rho} \phi \partial_{\mu} \partial_{\nu} h_{\rho \sigma} h^{\nu \sigma}+2 \partial_{\nu} \partial^{\rho} \phi \square h_{\rho \sigma} h^{\nu \sigma}-2 \partial_{\sigma} \partial_{\nu} \phi h_{\mu}^{\sigma} \partial^{\nu} \partial_{\rho} h^{\rho \mu}+2 \square \phi h_{\mu}^{\sigma} \partial_{\rho} \partial_{\sigma} h^{\rho \mu} \\
& +2 h\left(\partial_{\mu} \partial_{\nu} \phi \partial^{\sigma} \partial^{\mu} h_{\sigma}^{\nu}-\square \phi \partial_{\mu} \partial_{\nu} h^{\mu \nu}\right)-2 h \partial_{\mu} \partial_{\nu} \phi\left(\square h^{\mu \nu}-\partial_{\sigma} \partial^{\mu} h^{\nu \sigma}\right)-h\left(\partial_{\mu} \partial_{\nu} \phi \partial^{\mu} \partial^{\nu} h-\square \phi \square h\right),
\end{aligned}
$$

which is sufficient to show that any quantum mechanically generated loop correction involving vertices that come from the Gauss-Bonnet operator will not renormalize interactions of the form $(\partial \phi)^{2 n}$ at leading order in $M_{\mathrm{P}}$. Corrections instead come with at least an extra suppression in $1 / M_{\mathrm{P}}$.

\section{APPENDIX B: BLACK HOLES WITH GAUSS-BONNET HAIR}

In Ref. [7], an upper bound on the sGB coupling $\alpha$ has been derived under the assumption that the only operators in the scalar-tensor theory are given by

$$
\mathcal{L}=\frac{M_{\mathrm{P}}^{2}}{2} R-\frac{1}{2}\left(\partial_{\mu} \phi\right)^{2}+M_{\mathrm{P}} \alpha \phi \mathcal{R}_{\mathrm{GB}}^{2}
$$

In particular, requiring regularity of the second derivative of the scalar field at the horizon, the authors of Ref. [7] have shown that $\alpha<\alpha_{\max } \equiv r_{h}^{2} / \sqrt{192}$, where $r_{h}$ defines the position of the black hole horizon. In the following, we will show that this result is somehow fragile upon deformations of the theory (B1) and that the bound can indeed be relaxed if other operators become relevant in the vicinity of the black hole. For simplicity, let us assume that the theory near the horizon is dominated by the following operators, ${ }^{14}$

$\mathcal{L}=\frac{M_{\mathrm{P}}^{2}}{2} R-\frac{1}{2}\left(\partial_{\mu} \phi\right)^{2}+\beta\left(\partial_{\mu} \phi\right)^{2} \frac{\square \phi}{\Lambda^{3}}+M_{\mathrm{P}} \alpha \phi \mathcal{R}_{\mathrm{GB}}^{2}$,

where we included the cubic Galileon with coupling $\beta$. Let us parametrize the background metric as follows,

$\mathrm{d} s^{2}=-\mathrm{e}^{A(r)} \mathrm{d} t^{2}+\mathrm{e}^{B(r)} \mathrm{d} r^{2}+r^{2}\left(\mathrm{~d} \theta^{2}+\sin ^{2} \theta \mathrm{d} \varphi^{2}\right)$,

and let $r_{h}$ be the horizon, such that $\left.\mathrm{e}^{A}\right|_{r \rightarrow r_{h}^{+}} \rightarrow 0$ and $\left.A^{\prime}\right|_{r \rightarrow r_{h}^{+}} \rightarrow+\infty$. Solving the (rr)-component of the Einstein equations for $B(r)$, in the horizon limit $r \rightarrow r_{h}^{+}$, one finds that

\footnotetext{
${ }^{14}$ In fact, this assumption turns out to be quite general. One can try to consider the more general case of the theory (6) with the function $G_{3}(X)$ in the form for instance of an arbitrary polynomial of $X$. It turns out that the expression for $\phi^{\prime \prime}$ near the horizon is dominated by the lowest powers of $X$, leading therefore to the same bound (B6) that we find in the case (B2).
} 


$$
\left.\left.\mathrm{e}^{B}\right|_{r \rightarrow r_{h}^{+}} \rightarrow\left(r_{h}+\frac{4 \alpha \phi^{\prime}}{M_{\mathrm{P}}}\right) A^{\prime}\right|_{r \rightarrow r_{h}^{+}},
$$

at leading order in $\left.A^{\prime}\right|_{r \rightarrow r_{h}^{+}}$. The presence of the horizon requires that $\mathrm{e}^{B}$ diverges, which translates into the condition $M_{\mathrm{P}} r_{h}+4 \alpha \phi^{\prime}\left(r_{h}\right)>0$. Then, one can plug the result (B4) into the expression for $\phi^{\prime \prime}$, which can be obtained for instance from the scalar equation of motion, derived from (B2). In the horizon limit, the result takes on the form

$$
\begin{aligned}
\left.\phi^{\prime \prime}\right|_{r \rightarrow r_{h}^{+}} \rightarrow & -\frac{12 \alpha M_{\mathrm{P}}+r_{h}^{3} \phi^{\prime}+r_{h}^{2} \phi^{\prime 2}\left(\frac{4 \alpha}{M_{\mathrm{P}}}-\frac{\beta}{\Lambda^{3}}\right)}{\Lambda^{3} M_{\mathrm{P}}\left(r_{h}^{4}-96 \alpha^{2}\right)-2 r_{h}^{3} \phi^{\prime}\left(\beta M_{\mathrm{P}}-2 \alpha \Lambda^{3}\right)} \\
& \left.\cdot\left(M_{\mathrm{P}} r_{h}+4 \alpha \phi^{\prime}\right) A^{\prime}\right|_{r \rightarrow r_{h}^{+}} .
\end{aligned}
$$

Thus, in order for $\phi^{\prime \prime}$ to be finite at the horizon, the numerator in (B5) needs to vanish in the limit $r \rightarrow r_{h}^{+}$. Solving for $\phi^{\prime}\left(r_{h}\right)$, one finds two solutions, which are real only if

$$
r_{h}^{4}-192 \alpha^{2}+48 \beta \frac{M_{\mathrm{P}} \alpha}{\Lambda^{3}}>0
$$

Setting $\beta=0$, one immediately recovers the upper bound $\alpha_{\max }=r_{h}^{2} / \sqrt{192}$ of [7]. If instead $\beta \neq 0$, Eq. (B6) allows a wider range of values for the coupling $\alpha$, provided that $\alpha \beta>0$. Indeed, assuming $\beta \sim \mathcal{O}(1)$ and $r_{h}$ in the range of values of standard LIGO/Virgo and LISA black holes, $\alpha$ can now be as large as $M_{\mathrm{P}} / \Lambda^{3}$, which for $\Lambda \sim 10^{2} \mathrm{~km}^{-1}$ corresponds to $\alpha_{\max } \sim 10^{32} \mathrm{~km}^{2}$.

\section{APPENDIX C: DIMENSIONAL ESTIMATES FOR STATIC AND SPHERICALLY SYMMETRIC BACKGROUNDS}

Here, we present a dimensional estimate of the typical curvature $\mathcal{R}$ outside static spherically symmetric object of mass $M_{*}$, assuming the unperturbed GR solution. Since in vacuum both the Ricci tensor and the Ricci scalar are zero, we must look at the full Riemann tensor. A nonvanishing scalar we can build is the Kretschmann scalar,

$$
R_{\mu \nu \rho \sigma} R^{\mu \nu \rho \sigma} \simeq \frac{r_{s}^{2}}{r^{6}}
$$

where we evaluated for the Schwarzschild metric and $r_{s}=$ $M_{*} / M_{\mathrm{P}}{ }^{2}$ is the Schwarzschild radius. From this quantity, we can then give an estimate of the typical curvature as

$$
\mathcal{R}=\sqrt{R_{\mu \nu \rho \sigma} R^{\mu \nu \rho \sigma}} \sim \frac{r_{s}}{r^{3}} .
$$

The Gauss-Bonnet invariant instead, in vacuum, is precisely given by the Kretschmann scalar, and therefore

$$
\mathcal{R}_{\mathrm{GB}}^{2} \simeq \frac{r_{s}^{2}}{r^{6}} .
$$

As a worked example, consider the derivation of (16). Perturbing the operator $M_{\mathrm{P}} \alpha \phi \mathcal{R}_{\mathrm{GB}}^{2}$ around a Schwarzschild background, we obtain the following quadratic kinetic mixing (modulo numerical factors),

$$
M_{\mathrm{P}} \alpha \phi \mathcal{R}_{\mathrm{GB}}^{2} \rightarrow M_{\mathrm{P}} \alpha R_{\mu \nu \rho \sigma} \partial^{\mu} \pi \partial^{\sigma} h^{\nu \rho},
$$

where the Riemann tensor is taken to be evaluated on the background. Upon normalizing the graviton perturbation $\left(h \rightarrow h_{c} / M_{P}\right)$ and explicitly substituting the background scaling for the Riemann tensor (C1), we then find

$$
M_{\mathrm{P}} \alpha R_{\mu \nu \rho \sigma} \partial^{\mu} \pi \partial^{\sigma} h^{\nu \rho} \sim \alpha \frac{r_{s}}{r^{3}} \partial h_{c} \partial \pi
$$

reproducing the result of (16). Similarly consider the mixing induced by a HD operator, e.g., the cubic Galileon operator $\Lambda_{2}^{4} X Z$, i.e., $n=1, m=1$ in the notation of (11). From such a term, we schematically obtain a mixing,

$$
\begin{aligned}
\frac{1}{\Lambda^{3}}\left(\partial \phi_{0}\right)^{2} \partial \pi \partial h & =\frac{1}{\Lambda_{2}^{4}}\left(\frac{\Lambda_{3}}{\Lambda}\right)^{3}\left(\partial \phi_{0}\right)^{2} \partial \pi \partial h_{c} \\
& =\left(\frac{\Lambda_{3}}{\Lambda}\right)^{3} X_{0} \partial \pi \partial h_{c} \sim \alpha \frac{r_{s}^{2}}{r^{4}} \partial \pi \partial h_{c}
\end{aligned}
$$

where we have used (14) to solve for $X_{0}$ in the final step and reproduced (17).

\section{APPENDIX D: KINETIC MIXING FROM COSMOLOGY}

Consider the Lagrangian,

$$
\mathcal{L}=M_{\mathrm{P}}^{2} R+\Lambda_{2}^{4} X^{n} Z^{m},
$$

with $m=0,1,2,3$ and $n \geq 1$ and $Z=\frac{\partial^{2} \phi}{\Lambda^{3}}$. The HD operators $(m \geq 1)$, when expanded around some background solution with $X_{0}$ and $Z_{0}$, will generically induce a mixing of the form

$$
\Lambda_{2}^{4} X^{n} Z^{m} \supset \frac{\Lambda_{2}^{4}}{\Lambda^{3} M_{\mathrm{P}}} X_{0}^{n} Z_{0}^{m-1} \partial h_{c} \partial \pi \equiv \mathcal{Z}_{\mathrm{mix}}^{H} \partial h_{c} \partial \pi
$$

where $\Lambda_{2}^{2}=M_{\mathrm{P}} H_{0}$. On the other hand, the kinetic term for $\pi$ generically also receives a contribution,

$$
\Delta \mathcal{Z}_{\pi} \sim X_{0}^{n-1} Z_{0}^{m}
$$

On the cosmological background, we have $X_{0} \sim 1$, and $Z_{0} \sim \Lambda_{3}^{3} / \Lambda^{3}$, such that the mixing term and the new contribution to the kinetic term satisfy 


$$
\mathcal{Z}_{\text {mix }}^{H} \sim \Delta \mathcal{Z}_{\pi} \sim\left(\frac{\Lambda_{3}}{\Lambda}\right)^{3 m} \ll 1
$$

where $\Lambda_{3}^{3}=M_{\mathrm{P}} H_{0}^{2}$. Now, after diagonalizing and canonically normalizing, there is an induced coupling with matter of the form

$$
\frac{1}{M_{\mathrm{P}}} h_{\mu \nu}^{c} T^{\mu \nu} \rightarrow \frac{1}{M_{\mathrm{P}}} \frac{\mathcal{Z}_{\mathrm{mix}}^{H}}{\sqrt{\mathcal{Z}_{\pi}}} \pi_{c} T+\ldots
$$

Assuming there is a standard kinetic term for $\pi$ to begin with, we have $\mathcal{Z}_{\pi}=1+\Delta \mathcal{Z}_{\pi} \simeq 1$, and then the screening effect can be neglected. Furthermore, if there is at least one operator with $m \geq 1$, the induced coupling is at most of order

$$
\left(\frac{\Lambda_{3}}{\Lambda}\right)^{3} \pi T
$$

[1] B. P. Abbott et al. (LIGO Scientific and Virgo Collaborations), GWTC-1: A Gravitational-Wave Transient Catalog of Compact Binary Mergers Observed by LIGO and Virgo during the First and Second Observing Runs, Phys. Rev. X 9, 031040 (2019).

[2] C. A. R. Herdeiro and E. Radu, Asymptotically flat black holes with scalar hair: a review, Int. J. Mod. Phys. D 24, 1542014 (2015).

[3] M.S. Volkov, Hairy black holes in the XX-th and XXI-st centuries, in Proceedings, 14th Marcel Grossmann Meeting: Rome, Italy, 2015 (World Scientific, Singapore, 2017), Vol. 2, pp. 1779-1798.

[4] O. J. Tattersall, P. G. Ferreira, and M. Lagos, General theories of linear gravitational perturbations to a Schwarzschild black hole, Phys. Rev. D 97, 044021 (2018).

[5] O. J. Tattersall and P. G. Ferreira, Quasinormal modes of black holes in Horndeski gravity, Phys. Rev. D 97, 104047 (2018).

[6] T. P. Sotiriou and S.-Y. Zhou, Black Hole Hair in Generalized Scalar-Tensor Gravity, Phys. Rev. Lett. 112, 251102 (2014).

[7] T. P. Sotiriou and S.-Y. Zhou, Black hole hair in generalized scalar-tensor gravity: An explicit example, Phys. Rev. D 90, 124063 (2014).

[8] D. Ayzenberg and N. Yunes, Slowly-rotating black holes in Einstein-Dilaton-Gauss-Bonnet gravity: Quadratic order in spin solutions, Phys. Rev. D 90, 044066 (2014); Erratum, Phys. Rev. D 91, 069905 (2015).

[9] A. Maselli, H. O. Silva, M. Minamitsuji, and E. Berti, Slowly rotating black hole solutions in Horndeski gravity, Phys. Rev. D 92, 104049 (2015).

[10] R. Benkel, T. P. Sotiriou, and H. Witek, Dynamical scalar hair formation around a Schwarzschild black hole, Phys. Rev. D 94, 121503(R) (2016).

[11] G. Antoniou, A. Bakopoulos, and P. Kanti, Evasion of NoHair Theorems and Novel Black-Hole Solutions in GaussBonnet Theories, Phys. Rev. Lett. 120, 131102 (2018).

[12] J. L. Blàzquez-Salcedo, Z. Altaha Motahar, D. D. Doneva, F. S. Khoo, J. Kunz, S. Mojica, K. V. Staykov, and S. S. Yazadjiev, Quasinormal modes of compact objects in alternative theories of gravity, Eur. Phys. J. Plus 134, 46 (2019).
[13] H. Witek, L. Gualtieri, P. Pani, and T. P. Sotiriou, Black holes and binary mergers in scalar Gauss-Bonnet gravity: Scalar field dynamics, Phys. Rev. D 99, 064035 (2019).

[14] A. Nicolis, R. Rattazzi, and E. Trincherini, The Galileon as a local modification of gravity, Phys. Rev. D 79, 064036 (2009).

[15] D. Pirtskhalava, L. Santoni, E. Trincherini, and F. Vernizzi, Weakly broken Galileon symmetry, J. Cosmol. Astropart. Phys. 09 (2015) 007.

[16] G. W. Horndeski, Second-order scalar-tensor field equations in a four-dimensional space, Int. J. Theor. Phys. 10, 363 (1974).

[17] L. Santoni, E. Trincherini, and L. G. Trombetta, Behind Horndeski: structurally robust higher derivative EFTs, J. High Energy Phys. 08 (2018) 118.

[18] B. P. Abbott et al. (LIGO Scientific, Virgo, Fermi-GBM, and INTEGRAL Collaborations), Gravitational waves and Gamma-rays from a binary neutron star merger: GW170817 and GRB 170817A, Astrophys. J. 848, L13 (2017).

[19] C. de Rham and S. Melville, Gravitational Rainbows: LIGO and Dark Energy at its Cutoff, Phys. Rev. Lett. 121, 221101 (2018).

[20] T. Baker, E. Bellini, P. G. Ferreira, M. Lagos, J. Noller, and I. Sawicki, Strong Constraints on Cosmological Gravity from GW170817 and GRB 170817A, Phys. Rev. Lett. 119, 251301 (2017).

[21] P. Creminelli and F. Vernizzi, Dark Energy after GW170817 and GRB170817A, Phys. Rev. Lett. 119, 251302 (2017).

[22] J. M. Ezquiaga and M. Zumalacarregui, Dark Energy after GW170817: Dead Ends and the Road Ahead, Phys. Rev. Lett. 119, 251304 (2017).

[23] J. Sakstein and B. Jain, Implications of the Neutron Star Merger GW170817 for Cosmological Scalar-Tensor Theories, Phys. Rev. Lett. 119, 251303 (2017).

[24] P. Creminelli, M. Lewandowski, G. Tambalo, and F. Vernizzi, Gravitational wave decay into dark energy, J. Cosmol. Astropart. Phys. 12 (2018) 025.

[25] P. Creminelli, G. Tambalo, F. Vernizzi, and V. Yingcharoenrat, Dark-energy instabilities induced by gravitational waves, arXiv:1910.14035. 
[26] A. Adams, N. Arkani-Hamed, S. Dubovsky, A. Nicolis, and R. Rattazzi, Causality, analyticity and an IR obstruction to UV completion, J. High Energy Phys. 10 (2006) 014.

[27] B. Bellazzini, M. Lewandowski, and J. Serra, Amplitudes' Positivity, Weak Gravity Conjecture, and Modified Gravity, Phys. Rev. Lett. 123, 251103 (2019).

[28] C. de Rham, S. Melville, A. J. Tolley, and S.-Y. Zhou, Massive Galileon positivity bounds, J. High Energy Phys. 09 (2017) 072.

[29] B. Bellazzini, F. Riva, J. Serra, and F. Sgarlata, Beyond Positivity Bounds and the Fate of Massive Gravity, Phys. Rev. Lett. 120, 161101 (2018).

[30] C. de Rham, S. Melville, and A. J. Tolley, Improved positivity bounds and massive gravity, J. High Energy Phys. 04 (2018) 083.

[31] C. Armendariz-Picon, V. F. Mukhanov, and P. J. Steinhardt, A Dynamical Solution to the Problem of a Small Cosmological Constant and Late Time Cosmic Acceleration, Phys. Rev. Lett. 85, 4438 (2000).

[32] C. Armendariz-Picon, V. F. Mukhanov, and P. J. Steinhardt, Essentials of k essence, Phys. Rev. D 63, 103510 (2001).

[33] C. Deffayet, X. Gao, D. A. Steer, and G. Zahariade, From k-essence to generalised Galileons, Phys. Rev. D 84, 064039 (2011).

[34] J. Gleyzes, D. Langlois, F. Piazza, and F. Vernizzi, Healthy Theories Beyond Horndeski, Phys. Rev. Lett. 114, 211101 (2015).

[35] M. Zumalacárregui and J. Garcia-Bellido, Transforming gravity: from derivative couplings to matter to second-order scalar-tensor theories beyond the Horndeski Lagrangian, Phys. Rev. D 89, 064046 (2014).

[36] L. Hui and A. Nicolis, No-Hair Theorem for the Galileon, Phys. Rev. Lett. 110, 241104 (2013).

[37] E. Berti, K. Yagi, H. Yang, and N. Yunes, Extreme gravity tests with gravitational waves from compact binary coalescences: (II) Ringdown, Gen. Relativ. Gravit. 50, 49 (2018).

[38] E. Berti, V. Cardoso, L. Gualtieri, M. Horbatsch, and U. Sperhake, Numerical simulations of single and binary black holes in scalar-tensor theories: circumventing the no-hair theorem, Phys. Rev. D 87, 124020 (2013).

[39] L. Barack et al., Black holes, gravitational waves and fundamental physics: a roadmap, Classical Quantum Gravity 36, 143001 (2019).

[40] J. L. Blázquez-Salcedo, C. F. B. Macedo, V. Cardoso, V. Ferrari, L. Gualtieri, F. S. Khoo, J. Kunz, and P. Pani, Perturbed black holes in Einstein-dilaton-Gauss-Bonnet gravity: Stability, ringdown, and gravitational-wave emission, Phys. Rev. D 94, 104024 (2016).

[41] O. J. Tattersall and P. G. Ferreira, Forecasts for low spin black hole spectroscopy in Horndeski gravity, Phys. Rev. D 99, 104082 (2019).

[42] J. Khoury, Theories of dark energy with screening mechanisms, arXiv:1011.5909.

[43] E. Babichev and C. Deffayet, An introduction to the Vainshtein mechanism, Classical Quantum Gravity 30, 184001 (2013).

[44] B. P. Abbott et al. (LIGO Scientific and Virgo Collaborations), GW151226: Observation of Gravitational Waves from a 22-Solar-Mass Binary Black Hole Coalescence, Phys. Rev. Lett. 116, 241103 (2016).

[45] B. P. Abbott et al. (LIGO Scientific and Virgo Collaborations), GW170814: A Three-Detector Observation of Gravitational Waves from a Binary Black Hole Coalescence, Phys. Rev. Lett. 119, 141101 (2017).

[46] J. G. Williams, S. G. Turyshev, and D. H. Boggs, Progress in Lunar Laser Ranging Tests of Relativistic Gravity, Phys. Rev. Lett. 93, 261101 (2004).

[47] S. M. Merkowitz, Tests of gravity using Lunar laser ranging, Living Rev. Relativity 13, 7 (2010).

[48] K. Yagi, A new constraint on scalar Gauss-Bonnet gravity and a possible explanation for the excess of the orbital decay rate in a low-mass x-ray binary, Phys. Rev. D 86, 081504(R) (2012).

[49] N. Yunes, K. Yagi, and F. Pretorius, Theoretical physics implications of the binary black-hole mergers GW150914 and GW151226, Phys. Rev. D 94, 084002 (2016).

[50] C. de Rham, A. J. Tolley, and D. H. Wesley, Vainshtein mechanism in binary Pulsars, Phys. Rev. D 87, 044025 (2013).

[51] F. Dar, C. De Rham, J.T. Deskins, J. T. Giblin, and A. J. Tolley, Scalar gravitational radiation from binaries: Vainshtein mechanism in time-dependent systems, Classical Quantum Gravity 36, 025008 (2019).

[52] G. Franciolini, L. Hui, R. Penco, L. Santoni, and E. Trincherini, Effective field theory of black hole quasinormal modes in scalar-tensor theories, J. High Energy Phys. 02 (2019) 127.

[53] M. Punturo et al., The Einstein telescope: A third-generation gravitational wave observatory, Classical Quantum Gravity 27, 194002 (2010).

[54] T. Kobayashi, M. Yamaguchi, and J. Yokoyama, Generalized G-inflation: Inflation with the most general second-order field equations, Prog. Theor. Phys. 126, 511 (2011). 